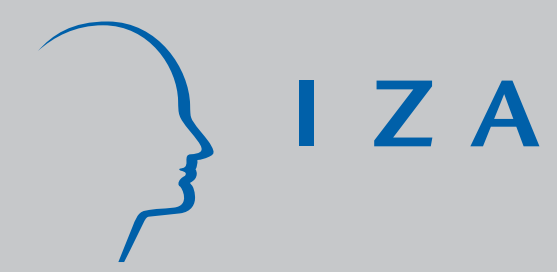

IZA DP No. 3825

The Effect of Active Labor Market Programs on Not-Yet Treated Unemployed Individuals

Gerard J . van den Berg

Annette Bergemann

Marco Caliendo

November 2008 


\title{
The Effect of Active Labor Market Programs on Not-Yet Treated Unemployed Individuals
}

\author{
Gerard J. van den Berg \\ VU University Amsterdam, IFAU-Uppsala, \\ IFS, Netspar, CEPR and IZA \\ Annette Bergemann \\ VU University Amsterdam and IZA \\ Marco Caliendo \\ IZA
}

\author{
Discussion Paper No. 3825 \\ November 2008
}

\author{
IZA \\ P.O. Box 7240 \\ 53072 Bonn \\ Germany \\ Phone: +49-228-3894-0 \\ Fax: +49-228-3894-180 \\ E-mail: iza@iza.org
}

\begin{abstract}
Any opinions expressed here are those of the author(s) and not those of IZA. Research published in this series may include views on policy, but the institute itself takes no institutional policy positions.

The Institute for the Study of Labor (IZA) in Bonn is a local and virtual international research center and a place of communication between science, politics and business. IZA is an independent nonprofit organization supported by Deutsche Post World Net. The center is associated with the University of Bonn and offers a stimulating research environment through its international network, workshops and conferences, data service, project support, research visits and doctoral program. IZA engages in (i) original and internationally competitive research in all fields of labor economics, (ii) development of policy concepts, and (iii) dissemination of research results and concepts to the interested public.
\end{abstract}

IZA Discussion Papers often represent preliminary work and are circulated to encourage discussion. Citation of such a paper should account for its provisional character. A revised version may be available directly from the author. 
IZA Discussion Paper No. 3825

November 2008

\section{ABSTRACT \\ The Effect of Active Labor Market Programs on Not-Yet Treated Unemployed Individuals*}

Labor market programs may affect unemployed individuals' behavior before they enroll. Such ex ante effects are hard to identify without model assumptions. We develop a novel method that relates self-reported perceived treatment rates and job-search behavioral outcomes, like the reservation wage, to each other, among newly unemployed workers. Job search theory is used to derive theoretical predictions. To deal with effect heterogeneity and selectivity, the effects of interest are estimated by propensity score matching. We apply the method to the German ALMP system, using a novel data set including self-reported assessments of the variables of interest as well as an unusually detailed amount of information on behavior, attitudes, and past outcomes. We find that the system generates a negative ex ante effect on the reservation wage and a positive effect on search effort.

JEL Classification: J64, C21, D83, D84

Keywords: policy evaluation, reservation wage, search effort, expectations, unemployment duration, program evaluation, active labor market policy, identification

Corresponding author:

Gerard J. van den Berg

Department of Economics

VU University Amsterdam

De Boelelaan 1105

1081 HV Amsterdam

The Netherlands

E-mail: gjvdberg@xs4all.nl

\footnotetext{
* We thank Armin Falk, Per Johansson, Gregory Jolivet, Peter Nilsson, and participants in the EEA 2008 Invited Session "Recent Developments in Program Evaluation" and in a June 2008 workshop at IZA (Bonn) for useful suggestions. We thank our colleagues at IZA for help with the data. The IAB (Nuremberg) kindly gave us permission to use the data. Caliendo thanks the German Research Foundation (DFG) for financial support of the project CA 829/1-1.
} 


\section{Introduction}

Labor market programs are common tools to help unemployed individuals move into work. Typically, a certain amount of time in unemployment elapses before individuals move into a program, and this means that most likely there is a time interval between the moment the agent realizes that he may be exposed to the policy and the actual exposure or treatment. This in turn means that the mere existence or availability of a program can have effects on the unemployed individuals' behavior before they actually move into the program. In fact, before the treatment occurs, the agent has an incentive to acquire information on the determinants of the process leading to treatment. After all, the probability of exposure to treatment is a determinant of the optimal strategy, and the more the agent knows about it, the better he can fine-tune his behavior in response to this, and the higher his expected present value will be. The agent's strategy will in turn affect the outcome of interest. As an example, consider a job search assistance program that helps the unemployed worker to search for jobs more efficiently for a given search effort. It makes sense for the individual to find out at which rate he will enter this program: the higher this rate, the more attractive it is to postpone search until the assistance has started. This amounts to a higher optimal reservation wage and a lower transition rate to work. $^{1}$

It is often relevant to know whether the overall effect of a program on outcomes like the duration of unemployment or the probability of employment within one year after entry into unemployment is driven by "ex ante" effects or by "ex post" effects (i.e. due to actual participation). If the ex post effect of having been trained on the exit rate to work is positive, whereas the ex ante effect on this rate is negative, then this may suggest that the program should be offered more frequently or earlier in the spell of unemployment. In this case, a comparison of a market where the program is offered to a market where it is not offered may just indicate that the net effect is zero. If the ex ante effect is positive, then one may inform the potential participants that they are eligible for participation. Knowing ex ante effects is also necessary to address whether programs affect the voluntary inflow into unemployment.

However, the existence of ex ante effects is hard to assess with commonly available data, and this hampers policy evaluation. The main problem is that "ex ante" effects are usually not identified - precisely because (i) instead of comparing outcomes by easily observable treatment statuses it involves comparing outcomes by treatment rates which are not directly observed, and (ii) all variation in treatment rate determinants that is observable to the researcher is typically also observable to the agent, and the agent will use this information in his behavior towards the

\footnotetext{
${ }^{1}$ See Van den Berg (2007) for an economic model of information acquisition about the probability of a future treatment, in a dynamic setting with forward-looking agents.
} 
outcome of interest. Empirical studies sometimes acknowledge that ex ante effects may exist but that they are not estimated. The ex post effect of treatment is then defined as the change in outcome relative to the outcome if treatment occurs later (see Abbring and Van den Berg, 2003, for an exposition).

A few studies have examined ex ante effects in specific settings. Black et al. (2003) use locally randomized assignment of treatment status to empirically examine whether this affects the voluntary inflow into the unemployment insurance (UI) regime. Here, the treatment regime starts right after entering the UI regime. Abbring and Van den Berg (2005) show that if the moment of treatment has a random element and if the observed treatment and labor market outcomes are duration variables, and if there is randomized variation in the treatment intensity, then identification of ex ante effects still requires a semi-parametric model structure and absence of anticipation of the moment of treatment (that is, no anticipation beyond what is captured in the treatment assignment equation; see Rosholm and Svarer, 2008, for an application). De Giorgi (2005) and Van den Berg, Bozio and Costa Dias (2008) use a policy discontinuity in time to study the effect of a treatment at 6 months unemployment duration on the probability finding work before 6 months. Specifically, he compares a situation where individuals in the inflow are aware of the policy to a situation where the policy regime has not yet been introduced. Lalive, Van Ours and Zweimüller (2005) observe whether and when unemployed individuals receive advance warnings about the timing of future treatments. By viewing such warnings as treatments themselves, they can apply the semi-parametric timing-of-events framework of Abbring and Van den Berg (2003) to study their effect.

In this paper we develop and apply a novel general method to identify ex ante effects. Specifically, we identify ex ante effects of labor market programs by using self-reported variables of unemployed workers in a panel survey. They are asked about their perceived probability of being treated in future periods, and they are also asked about their current optimal job search strategy, notably their current reservation wage and their current search effort. All else equal, the expectation of a future event that increases the individual's expected present value should increase his reservation wage. For example, if an individual thinks it is likely that he will enter a training program, and if he thinks that the training program increases his chances to find work, then the present value of being unemployed is high, and consequently it does not make sense to accept low wages, so the current reservation wage is high. If we do not find effects on the current reservation wage or, in general, on current search effort, then there is no ex ante effect, and the transition rate to work equals the rate in absence of the program. Since the reservation wage is increasing in the expected present value of unemployment, our results also have implications for how the well-being of the unemployed depends on future program participation.

Our data are from the first wave of the IZA Evaluation Data Set (see Caliendo et 
al., 2008, for details). This in an ongoing data collection process in which an inflow sample of unemployed in Germany will be followed over time. In this study we only use information from the first survey wave and we do not use register information. The survey interviews were held in late 2007 and early 2008 among individuals who had shortly before that become unemployed. Respondents answered an extensive set of questions inter alia about their search behavior, reservation wages, previous employment experience, and expectations about program participation. Our estimation sample contains about 8,000 individuals who are asked the questions about their expectations of program participation and their reservation wage. ${ }^{2}$ Note that we do not use self-reported assessments of counterfactual outcomes at the individual level. We deal with individual heterogeneity by matching individuals with different outcomes on the basis of a propensity score for the subjective probability of treatment. Notice that in this setting the propensity score is the probability that the probability of treatment has a certain value, which is one level deeper than in the case of standard (ex post) treatment effect evaluation. A standard regression approach is less feasible because dynamic economic theory predicts that all determinants interact with the effects we are interested in, so effect heterogeneity can be expected to be a pivotal feature. Moreover, it is important that the "common support" condition is satisfied for the propensity score, to prevent that the results are driven by a comparison of individuals in different entitlement regimes. The data contain a number of self-reported personality and behavioral assessments and individual past labor market outcomes that allow for a rich set of conditioning variables in the matching procedure.

Clearly, the currently available data do not allow for a confrontation with actual outcomes of program participation, unemployment duration, and characteristics of the accepted job. Note that the question whether individuals correctly estimate the occurrence and benefits of future events is not of primary concern, because it is their perception of this that affects their current behavior. Of course, detection of a misperception of the treatment probability may have policy implications as well.

Our paper is organized as follows. In Section 2 we present a job search model framework to structure the analysis. We allow for the possibility that individuals like or dislike a treatment for reasons other than their effect on labor market outcomes. For example, training may help to find a job but may nevertheless lead to disutility because of the time that needs to be dedicated to participation in the program. If the probability of entering a program with positive ex post effects does not affect the current reservation wage then this implies that the treatment period carries a

\footnotetext{
${ }^{2}$ Self-reported assessments on attitudes, decisions and behavior have recently become popular in economics, but such data have since long been used in the literature on job search and unemployment duration. See for example Yoon (1981), Lancaster and Chesher (1983), and Van den Berg and Gorter (1997), for structural analyses and Lancaster (1985) for reduced-form analyses. Yoon (1981) uses search effort indicators while the others use reservation wage variables.
} 
disutility, and it may be useful to adjust the program design. In Section 3 we describe the data and the relevant self-reported variables. Section 4 contains the estimation results. Section 5 concludes.

\section{A job search model framework}

The basic idea behind our empirical approach is relatively obvious. As noted in the introduction, if an individual expects a beneficial treatment to take place with a high probability then this reduces the incentive to leave unemployment before that. However, to explain our approach in more detail, it is useful to develop a job search model. We start with a model in which the moment of treatment arrives according to a Poisson process. After that we explain that the main predictions also apply in more general models.

Consider an unemployed individual who searches sequentially for a job. Given a particular search effort $s$, job offers arrive according to the rate $\lambda s$. Offers are random drawings from a wage offer distribution $F(w)$. Every time an offer arrives the decision has to be made whether to accept it or to reject it and search further. Once a job is accepted, it will be held forever at the same wage. During unemployment, a flow of benefits $b$ is received (possibly including a non-pecuniary utility of being unemployed) and a flow of search $\operatorname{costs} c(s)$ has to be paid, where we take $c(s)=$ $\frac{1}{2} c_{0} s^{2}$. This is a conventional and convenient functional form (see e.g. Van den Berg and Van der Klaauw, 2006, and references therein). The individual maximizes the expected present value of income or utility over an infinite horizon.

Now let us introduce treatments or program participation in this framework. To be precise, we model the perception of the individual about the treatment rate and the treatment effect. These need not correspond to the actual rate and effect. For expositional reasons, we write this section as if the individual's perceptions and expectations are correct, to prevent that we always have to make the qualification that it is the perceptions that we are capturing. If the individual has not yet been treated, there is a rate $\eta \geq 0$ at which he will enter the treatment. The treatment can affect the parameter $\lambda$ of the job finding rate, and/or it can affect the wage offer distribution $F(w)$, or it can have no effect at all. The individual (thinks he) knows the effect of the treatment, but the only thing he perceives about the moment of entering the treatment is that it occurs at the rate $\eta$.

The expected present value before treatment is denoted by $R$. Upon treatment, the expected present value jumps by an amount $R_{p}-R-\gamma$ which can be positive or negative. Here, $R_{p}$ is the expected present value after the treatment and $\gamma$ is the direct cost of treatment. The sign of $\gamma$ depends on whether the individual expects to enjoy the treatment per se or not, and this also includes opportunity costs due to a possible "lock-in" time in the program. The gain $R_{p}-R$ due to the treatment effect 
on the individual labor market conditions can be positive or negative (see below). For simplicity we assume that the determinants $b, F, \lambda, c_{0}, \eta$ and the discount rate $\rho$ are constant as a function of unemployment duration for a given treatment status. This implies that the optimal strategy is constant over time until the moment of treatment. Using the familiar returns-to-assets representation of Bellman's equation we have

$$
\begin{aligned}
& \rho R=\max _{s} b-\frac{1}{2} c_{0} s^{2}+\lambda s \mathbb{E}_{F} \max \left\{\frac{w}{\rho}-R, 0\right\}+\eta\left(R_{p}-R-\gamma\right) \\
& \rho R_{p}=\max _{s_{p}} b-\frac{1}{2} c_{0} s_{p}^{2}+\lambda_{p} s_{p} \mathbb{E}_{F_{p}} \max \left\{\frac{w}{\rho}-R_{p}, 0\right\}
\end{aligned}
$$

where the expectations are taken over the wage offer distribution. Clearly, the optimal strategy of the unemployed individuals before treatment can be characterized by a reservation wage $\phi$, giving the minimal acceptable wage offer, and an optimal search effort (which we simply denote by $s$ ). Specifically, $\phi=\rho R$, and $s$ follows from:

$$
c_{0} \cdot s=\lambda \mathbb{E}_{F} \max \left\{\frac{w}{\rho}-R, 0\right\}
$$

Suppose $\gamma=0$. Then $R_{p}-R$ is positive if $\lambda_{p}>\lambda$ and if $F_{p}$ first-order stochastically dominates $F$.

Let $G$ denote the total gain $R_{p}-R-\gamma$ due to the treatment, and let us explicate the dependence of $\phi$ and $s$ on $\eta$ and $G$ by writing $\phi(\eta, G)$ and $s(\eta, G)$. Then, for a given policy with values $\eta=\eta_{0}$ and parameter values $\lambda_{p}$ and $F_{p}$ leading to $G=G_{0}$, we can define the ex ante effects as $\phi\left(\eta_{0}, G_{0}\right)-\phi(0,0)$ and $s\left(\eta_{0}, G_{0}\right)-s(0,0)$. A number of results follow directly from the model. ${ }^{3}$ For sake of exposition we ignore for the moment non-interior optimal solutions and limiting cases. If $G>0$ and $\eta>0$ then the ex ante effect on $\phi$ is positive, while on $s$ it is negative, meaning that $\phi$ is larger and $s$ is smaller than in the case in which the program is totally absent (or, more precisely, the individual is not aware of the program). In other words, if the expected gain and the treatment rate are positive then this implies more selective behavior and less search effort. Both of these serve to reduce the transition rate $\theta$ from unemployment to employment before treatment, which equals $\theta=\lambda s(1-F(\phi))$. If $G<0$ and $\eta>0$ then the ex ante effects on $\phi$ and $s$ are negative and positive, respectively. An observed difference between the reservation wage (or the search effort) among those who report some particular $\eta>0$, and otherwise equivalent individuals who report $\eta=0$, captures for that value of $\eta$ the ex ante effect and its sign, and from this we can infer the sign of $G$.

\footnotetext{
${ }^{3}$ These can be proven by substituting $\phi=\rho R$ and equations (2) and (3) into equation (1) and subsequent differentiation.
} 
More generally, if $G>0$ then $d \phi / d \eta>0$ and $d s / d \eta<0$, whereas if $G<0$ then the opposite applies, and if $G=0$ then $d \phi / d \eta=d s / d \eta=0 .{ }^{4}$ Thus, the empirical signs of $d \phi / d \eta$ and $d s / d \eta$ can be used to infer whether $G \gtrless 0$. If the empirical signs of $d \phi / d \eta$ and $d s / d \eta$ are zero then there are no ex ante effects, and we infer that $G=0$, so either the program is ineffective, or the program is beneficial but the individual dislikes the treatment itself. Note that the ex ante effects of $\eta$ on $\phi$ and $s$ always have opposite signs regardless of whether $G \gtrless 0$. With data on $\eta, \phi$ and $s$, this is a testable theoretical implication.

The derivatives $d \phi / d \eta$ and $d s / d \eta$ can be shown to depend on all other model determinants, so $\eta$ interacts with all other model determinants in its effects on $\phi$ and $s$. This is not surprising. The importance of the treatment entry rate captures the extent to which the treatment leads to an improvement in the expected present value. The model probability of actually undergoing the treatment is $\eta /(\eta+\theta)$. This means that model determinants leading to a high value of $\theta$ reduce by way of this chain the magnitude of the ex ante effects. Similarly, the relevance of the treatment is larger if the future is less heavily discounted. These insights suggest that ex ante effects are likely to be heterogeneous across agents. This makes the matching evaluation method particularly useful. In addition, the interactions suggest that it may be useful to include outcome determinants like the unemployment benefits level in the propensity score, and to also include labor market history indicators which capture variation in $\theta$.

Notice that extreme cases lead to absence of ex ante effects. For example, if $\rho=\infty$ or if $b$ sufficiently exceeds the upper bound of the support of $F$ and $F_{p}$, then the ex ante effects are zero for any value of $\eta, \gamma, \lambda$ and $\lambda_{p}$.

Clearly, the above results are very robust with respect to the model assumptions. For example, income maximization can be replaced by utility maximization, and the cost function $c(s)$ can be generalized to a large class of convex increasing functions. It is possible that individuals believe that they know more about the moment treatment than that it arrives according to a Poisson process. At the extreme, they behave in accordance with knowing that it will take place at the deterministic duration $T$. In that case, the appropriate job search model is a nonstationary model along the lines of Van den Berg (1990), and we can derive the same results as above. In particular, the ex ante effects are larger if the time interval until $T$ is smaller. There are also interactions in this case; for example the effects are larger if the exit rate to work is generally small and the discount rate is small. Other forms of nonstationarity, such as negative duration dependence of the benefits and the job offer arrival rate, can be incorporated as well.

\footnotetext{
${ }^{4}$ To derive this, note that $G:=R_{p}-R-\gamma$ itself depends on $\eta$ via $R$.
} 


\section{Data}

In the empirical analysis we estimate ex ante effects for newly unemployed workers of the comprehensive German package of active labor market policies (ALMP) for unemployment benefits recipients. The most prominent ALMP in Germany are short training programs and job search assistance programs. However, also start-up subsidies for the unemployed, job creation programs, long-term (re-)training programs and wage subsidies for jobs in the private sector are of quite considerable size (see Bernhard et al., 2008, for a recent overview). In Germany, as in other European countries, case workers have a large influence on the (timing of the) participation of an unemployed worker in ALMP. Newly unemployed individuals are typically assigned to job search assistance programs or training programs. Long term unemployed individuals are more often assigned to employment programs, consisting of either wage subsidy programs for jobs in the private sector or job creation programs.

The data we use are from the IZA Evaluation Data Set. As explained in Section 1, this survey data set targets an inflow sample into unemployment from June 2007 to May 2008. The key feature of the data set is that individuals are interviewed shortly after they become unemployed and are asked a variety of non-standard questions about attitudes and expectations (see Caliendo et al., 2008, for details). The sampling is restricted to individuals who are 16 to 54 years old, and who receive or are eligible to receive unemployment benefits under the German Social Code III. From the monthly unemployment inflows of approximately 206,000 individuals in the administrative records, a $9 \%$ random sample is drawn which constitutes the gross sample. Out of this gross sample each month representative samples of approximately 1,450 individuals are interviewed, so that after one year 12 monthly cohorts are gathered.

For the first wave 17,396 interviews have been realized and individuals are interviewed about two months after they become unemployed. We restrict our analysis to individuals who are still unemployed and actively search for a job. That is, we exclude individuals who have found a job already, participate in a program or do not search for other reasons. ${ }^{5}$ This leaves us with a preliminary sample of 8,612 individuals from which we further exclude the lowest and highest percentile of the reported hourly reservation wage and the reported benefit level as well as individuals with missing values for any key variables. This leaves us with a sample of 7,914 individuals. Table 1 gives some descriptive statistics. Note that $68 \%$ live in West Germany and 6\% do not have German citizenship. Regarding the education level, 25\% have a high school degree and $33 \%$ have no or a low degree. Before entering unemployment the majority of individuals was in regular employment (65\%). Individuals are asked questions regarding their "locus of control" which is a generalized expectancy about

\footnotetext{
${ }^{5}$ Of these three categories, program participation constitutes by far the smallest.
} 
internal versus external control of reinforcement (Rotter, 1966). Whereas persons whose external locus of control personality trait dominates believe that everything what happens is beyond their control, people with an internal locus of control are confident that outcomes are contingent on their decisions and behavior. ${ }^{6}$ The latter applies to $54 \%$ of our sample.

The key variable for our analysis, $\eta$, is measured by the answer to the question how likely it is that ALMP participation occurs conditional on remaining unemployed in the upcoming three months (see Figure 1). This explicitly merges all ALMP measures (the main ALMP for short-term unemployed workers are training, job search assistance, and subsidized work). The answers range from 0 ("very unlikely", which is reported by $22 \%$ of the individuals), 1, 2, etc. up to 10 ("very likely", which is reported by $17 \%$ ). For the analysis we construct a binary measure by grouping $0-4$ into the category " $\eta$-low" and $5-10$ into " $\eta$-high". The search effort $s$ is operationalized as the number of search channels used where the maximum number is 10. This is in line with e.g. Van den Berg and Van der Klaauw (2006) and references therein who also use this outcome as an indicator of search effort. The coefficients of variation of $\phi$ and $s$ are both equal to 0.33. Table A.1 in the Appendix shows some descriptives differentiated for the groups with high $\eta(4,576$ individuals) and low $\eta$ (3,338 individuals). The t-test results presnetd in this Table and also the regression results from $\eta$ on $X$ presented in Table A.2 show that people in West Germany and females have a higher $\eta$, whereas $\eta$ decreases with age and higher professional training.

\section{Empirical Implementation and Results}

We first carry out some basic regression analyses of $\log \phi$ and $s$ on $\eta$ and on explanatory variables, i.e. ${ }^{7}$ :

$$
\begin{aligned}
& \log \phi=\beta_{0}+\beta_{1} \eta+\beta_{2} X+\varepsilon \\
& s=\alpha_{0}+\alpha_{1} \eta+\alpha_{2} X+\varepsilon_{s}
\end{aligned}
$$

Next, and more importantly, we perform propensity score matching (introduced by Rosenbaum and Rubin, 1983; see, e.g., Caliendo and Kopeinig, 2008, and Imbens and Wooldridge, 2008, for recent overviews). For this we estimate the propensity score for the probability to be in the " $\eta$-high" category mentioned above. Then we

\footnotetext{
${ }^{6}$ Locus of control is measured by a set of statements to which individuals could reply on a scale of ' 1 ' (I do not agree at all) to '7' (I agree fully), e.g., 'How my life takes course is entirely dependent on me' or 'Success is gained through hard work'. We summed up the positive answers and build a single dummy variable if the answers exceeded a certain threshold. Later results are neither sensitive to the threshold nor to the exclusion of the dummy variable.

${ }^{7}$ These results are virtually identical to the matching results (see Table A.3 in the Appendix).
} 
estimate "ATET" where the "treatment" is the (indicator of the) perceived rate at which ALMP participation occurs in the near future. The set of conditioning variables $X$ is large and includes individual past labor market history outcomes, the current benefits level, indicators of region, gender, nationality, marital status, children, age, education, past training, month of entry, and the locus of control indicator (see Table A.4 in the Appendix for estimations results). Together these should take care of possible selection effects. Most of these are significant in the propensity score and we get a nice region of common support between both groups (see Figure A.1 for the propensity score distribution in both groups). We are not concerned about reverse causality issues over and above the selection captured through $X$. In particular, it is unlikely that program assignment causally depends on the individual's reservation wage. If assignment depends on search effort then most likely the sign of the reverse relationship between $s$ and $\eta$ is negative, so that a positive empirical relation may under-estimate the true causal effect we are interested in.

It turns out that the ex ante effects on the reservation wage and on the search effort are significantly negative and significantly positive, respectively (see Table 2). ${ }^{8}$ This means that individuals try to prevent program participation by accepting worse jobs and by searching harder than they would do if the programs were absent. If the actual program participation either has no ex post effect or improves the individuals' ex post labor market conditions, it follows that necessarily $\gamma>0$, i.e. that individuals dislike the actual participation. The regression results and the matching results are in full agreement. Indeed, the matching estimates are virtually equal to the corresponding regression coefficients, and all ex ante effects are significant in each analysis. The matching results are robust with respect to the variables in $X$ and the inclusion of higher-order terms and interactions in $X$. Notice also that the ex ante effects on $\phi$ and $s$ have opposite signs, which confirms the theory.

In the absence of duration and wage outcomes it is hard to estimate the ensuing effect on the exit rate to work $\theta$. A very conservative estimate is obtained by ignoring the increase in search effort and by assuming that the relevant individual wage offer distribution is the overall sample distribution of wages accepted by individuals who find a job in the two months before the interview. A decrease of $3 \%$ of the reservation wage (corresponding to an increase of the ALMP treatment rate from a typical low level to a typical high level) then leads to an increase of the fraction of acceptable wage offers by $8 \%$, and therefore to an increase of $\theta$ by $8 \%$. In reality the individual wage offer distribution is likely to be much less dispersed than the aggregate wage offer distribution and even less dispersed than the aggregate distribution of accepted

\footnotetext{
${ }^{8}$ Results are not sensitive to the chosen cut-off levels. Additionally, we also implemented the estimators for three groups, i.e., individuals with low (0-2), medium (3-7) and high (8-10) expectations. It turns out that there are no significant differences between the medium and high group, but individuals in the high and medium group have a significantly lower reservation wage when compared to the low group.
} 
wages, which would lead to a higher elasticity estimate.

\section{Conclusions}

Our approach to identify ex ante effects of active labor market policies or treatments does not require estimation of treatment rates from selective samples involving noanticipation assumptions, and neither does it require superior knowledge about (institutional features of) the assignment process. It is therefore generally applicable. We use the method to study ex ante effects of the comprehensive German package of active labor market policies for unemployment benefits recipients. We find that the ex ante effect on the reservation wage and search effort are negative and positive, respectively. This means that individuals try to prevent program participation, by accepting worse jobs and by searching harder than they would do if the programs were absent. We conjecture that this is to a large extent because individuals dislike the actual participation experience. The program package reduces the welfare of the newly unemployed. At the same time, observational studies that estimate average causal effects of the programs from actual participation, and that use the not-yet treated as a control group, would underestimate a positive causal effect of participation versus absence of the programs, by neglecting the indirect ex ante effect.

Our current data set is the first wave of a longitudinal survey and is therefore essentially cross-sectional. It is an interesting topic for further research to confront the variables that we use to actual outcomes of program participation, unemployment duration, and characteristics of the accepted job. 


\section{References}

Abbring, Jaap H. and Gerard J. van den Berg (2003), "The non-parametric identification of treatment effects in duration models", Econometrica, 71, 1491-1517.

Abbring, Jaap H. and Gerard J. van den Berg (2005), "Social experiments and instrumental variables with duration outcomes", Working paper, IZA, Bonn.

Bernhard, Sarah, Katrin Hohmeyer, Eva Jozwiak, Susanne Koch, Thomas Kruppe, Gesine Stephan and Joachim Wolff (2008), "Aktive Arbeitsmarktpolitik in Deutschland und ihre Wirkungen", Working paper, IAB, Nuremberg.

Black, Dan A., Jeffrey A. Smith, Mark C. Berger, and Brett J. Noel (2003), "Is the threat of reemployment services more effective than the services themselves? Evidence from random assignment in the UI system", American Economic Review, 93, 1313-1327.

Caliendo, Marco, Armin Falk, Lutz Kaiser, Hilmar Schneider, Arne Uhlendorff, Gerard J. van den Berg, and Klaus F. Zimmermann (2008), "The IZA Evaluation Data Set - The First Wave", Working Paper, Bonn.

Caliendo, Marco, and Sabine Kopeinig (2008), "Some practical guidance for the implementation of propensity score matching", Journal of Economic Surveys, 22, $31-72$.

De Giorgi, Giacomo (2005), "Long term effects of a mandatory multistage program: The New Deal for Young People in the UK", Working paper, UCL, London.

Imbens, Guido I. and Jeffrey M. Wooldridge (2008), "Recent developments in the econometrics of program evaluation", Journal of Economic Literature, forthcoming.

Lalive, Rafael, Jan C. van Ours, and Josef Zweimüller (2005), "The effect of benefit sanctions on the duration of unemployment", Journal of the European Economic Association, 3, 1386-1417.

Lancaster, Tony and Andrew D. Chesher (1983), "An econometric analysis of reservation wages", Econometrica, 51, 1661-1676.

Lancaster, Tony (1985), "Simultaneous equations models in applied search theory", Journal of Econometrics, 28, 155-169.

Leuven, Edwin, and Barbara Sianesi (2003): "PSMATCH2: Stata Module to Perform Full Mahalanobis and Propensity Score Matching, Common Support Graphing, and Covariate Imbalance Testing," Software, http://ideas.repec.org/c/boc/ bocode/s432001.html.

Rosenbaum, Paul R. and Donald B. Rubin (1983): "The central role of the propensity score in observational studies for causal effects", Biometrika, 70, 41-55. 
Rosholm, Michael and Michael Svarer (2008), "Estimating the threat effect of active labour market programmes", Scandinavian Journal of Economics, forthcoming.

Rotter, Julian B. (1966), Generalized expectancies for internal versus external control of reinforcement, American Psychological Association, Washington DC.

Silverman, Bernard W. (1986), Density Estimation for Statistics and Data Analysis, Chapman \& Hall, London.

Van den Berg, Gerard J. (1990), "Nonstationarity in job search theory", Review of Economic Studies, 57, 255-277.

Van den Berg, Gerard J. and Cees Gorter (1997), "Job search and commuting time", Journal of Business and Economic Statistics, 15, 269-281.

Van den Berg, Gerard J. and Bas van der Klaauw (2006), "Counseling and monitoring of unemployed workers: Theory and evidence from a controlled social experiment", International Economic Review, 47, 895-936.

Van den Berg, Gerard J. (2007), "An economic analysis of exclusion restrictions for instrumental variable estimation", Working paper, IZA, Bonn.

Van den Berg, Gerard J., Antoine Bozio and Monica Costa Dias (2008), "Policy discontinuity and duration outcomes", Working paper, VU University Amsterdam.

Yoon, Bong J. (1981), "A model of unemployment duration with variable search intensity", Review of Economics and Statistics, 63, 599-609. 


\section{Figures and Tables}

Table 1: Selected Descriptives for the Estimation Sample

\begin{tabular}{|c|c|}
\hline Variable & Mean \\
\hline Number of Observations & 7,914 \\
\hline West Germany & 0.68 \\
\hline Female & 0.51 \\
\hline Internal Locus of Control & 0.54 \\
\hline Age (in years) & $\begin{array}{r}35.55 \\
(10.58)\end{array}$ \\
\hline German Citizenship & 0.94 \\
\hline Married (or cohabiting) & 0.39 \\
\hline One Child & 0.19 \\
\hline Two (or more) Children & 0.14 \\
\hline \multicolumn{2}{|l|}{ School Leaving Degree } \\
\hline No degree & 0.03 \\
\hline Low & 0.30 \\
\hline Medium & 0.42 \\
\hline High & 0.25 \\
\hline \multicolumn{2}{|l|}{ Employment Status before Unemployment } \\
\hline Subsidized Employment & 0.07 \\
\hline School, Apprentice, Military, etc. & 0.15 \\
\hline Maternity Leave & 0.05 \\
\hline Regular Employment & 0.65 \\
\hline Other & 0.08 \\
\hline \multicolumn{2}{|l|}{ Professional Training } \\
\hline None & 0.10 \\
\hline Apprenticeship, External Training, etc. & 0.71 \\
\hline University Degree, Master Craftsman & 0.19 \\
\hline Unemployment Benefit Recipient (1=yes) & 0.78 \\
\hline Level of Benefits (in Euro/month) & $\begin{array}{r}691.17 \\
(328.10)\end{array}$ \\
\hline Reservation Wage (in Euro/hour) & $\begin{array}{r}7.50 \\
(2.50)\end{array}$ \\
\hline Number of Search Channel(s) Used & $\begin{array}{r}5.10 \\
(1.67)\end{array}$ \\
\hline
\end{tabular}

Source: IZA Evaluation Data Set, own calculations.

Note: All numbers are shares unless stated otherwise; standard deviation in parentheses. 
Figure 1: Subjective ALMP Participation
Probability

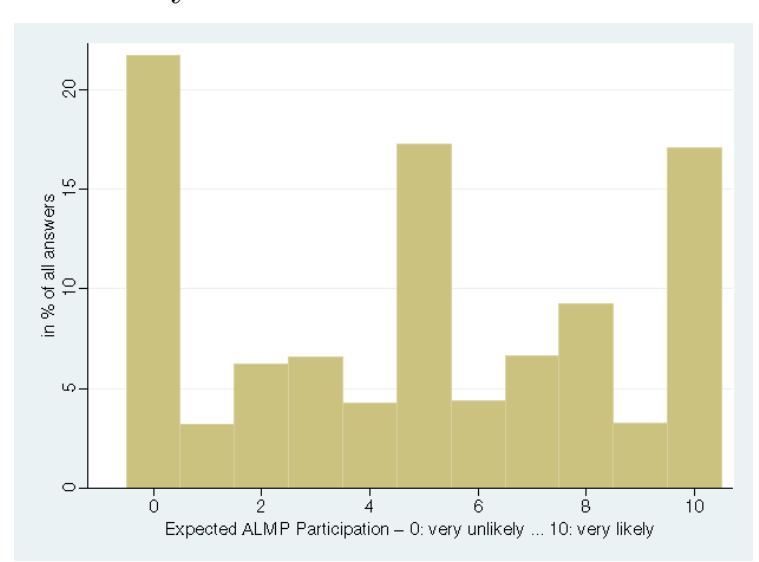

Note: Individuals where asked the following question: "Assume that you are still unemployed in the next three months. How likely is it that you participate in an ALMP program? Please use rates between ' 0 ' (very unlikely) to ' 10 ' (very likely)."

Table 2: Matching Results - Reservation Wage and Number of Search Channels

\begin{tabular}{lrrr}
\hline \hline Outcome Variable & Effect & s.e. & $\mathrm{t}$ \\
\hline Log Hourly Reservation Wage (in $€)$ & -0.030 & 0.007 & -4.063 \\
Number of search channels & 0.281 & 0.041 & 6.889 \\
\hline \hline
\end{tabular}

Note: We apply kernel (epanechnikov) matching with common support; for the bandwidth we follow Silverman's (1986) rule-of-thumb and use 0.06 . Standard errors are based on 100 bootstrap replications. Extensive sensitivity analyses are available on request by the authors; results are not sensitive to the kernel or bandwidth choice. Estimations are done using the PSMATCH2 package by Leuven and Sianesi (2003).

Matching Quality: The mean (median) standardized bias before matching is 4.93 (3.31), the according bias after matching is $0.61(0.44)$. 


\section{A Appendix}

Table A.1: Selected Descriptives - Differentiated by High/Low Participation Expectation

\begin{tabular}{|c|c|c|c|}
\hline Variable & $\eta=0-4$ & $\eta=5-10$ & p-value \\
\hline Number of Observations & 3,338 & 4,576 & \\
\hline West Germany & 0.64 & 0.71 & 0.00 \\
\hline Female & 0.49 & 0.52 & 0.02 \\
\hline Internal Locus of Control & 0.53 & 0.55 & 0.18 \\
\hline German citizenship & 0.95 & 0.94 & 0.00 \\
\hline Married (or cohabiting) & 0.41 & 0.38 & 0.00 \\
\hline No children & 0.67 & 0.66 & 0.65 \\
\hline One Child & 0.19 & 0.19 & 0.76 \\
\hline Two (or more) Children & 0.14 & 0.15 & 0.14 \\
\hline Age (in years) & $\begin{array}{r}36.53 \\
(10.50)\end{array}$ & $\begin{array}{r}34.83 \\
(10.59)\end{array}$ & 0.00 \\
\hline \multicolumn{4}{|l|}{ School Leaving Degree } \\
\hline No degree & 0.02 & 0.03 & 0.04 \\
\hline Low & 0.27 & 0.32 & 0.00 \\
\hline Medium & 0.42 & 0.42 & 0.96 \\
\hline High & 0.28 & 0.23 & 0.00 \\
\hline \multicolumn{4}{|l|}{ Professional Training } \\
\hline None & 0.09 & 0.11 & 0.00 \\
\hline Apprenticeship, External Training, etc. & 0.68 & 0.73 & 0.00 \\
\hline University Degree, Master Craftsman & 0.23 & 0.16 & 0.00 \\
\hline \multicolumn{4}{|l|}{ Employment status before Unemployment } \\
\hline Employed & 0.67 & 0.64 & 0.02 \\
\hline Subsidized Employment & 0.08 & 0.07 & 0.25 \\
\hline School, Apprentice, Military, etc. & 0.13 & 0.16 & 0.00 \\
\hline Maternity Leave & 0.04 & 0.05 & 0.16 \\
\hline Other & 0.08 & 0.08 & 0.27 \\
\hline Unemployment Benefit Recipient (yes) & 0.76 & 0.80 & 0.00 \\
\hline Reservation Wage (in Euro) & $\begin{array}{r}7.72 \\
(2.67)\end{array}$ & $\begin{array}{r}7.34 \\
(2.36)\end{array}$ & 0.00 \\
\hline Search Channel(s) (Number of) & $\begin{array}{r}4.94 \\
(1.68)\end{array}$ & $\begin{array}{r}5.21 \\
(1.65)\end{array}$ & 0.00 \\
\hline
\end{tabular}

Source: IZA Evaluation Data Set, own calculations.

Note: All numbers are shares unless stated otherwise; p-value refers to a two-sided t-test of mean equality between both groups. 
Table A.2: OLS Estimation Results - Expected Program Participation

\begin{tabular}{lc}
\hline \hline & $\eta$ \\
\hline West Germany & $0.546^{* * *}$ \\
Female & $0.325^{* * *}$ \\
Internal Locus of Control & $0.196^{* *}$ \\
German citizenship & $-.333^{*}$ \\
Married (or cohabiting) & $-.250^{* *}$ \\
No Children (Reference cat.) & \\
One Child & 0.124 \\
Two (or more) Children & $0.312^{* *}$ \\
Age (17-24 years) & \\
Age (25-34 years) & $-.318^{* *}$ \\
Age (35-44 years) & $-.309^{* *}$ \\
Age (45-55 years) & $-.565^{* * *}$ \\
School Leaving Degree: No degree (Ref.) & \\
Low & -.175 \\
Medium & -.189 \\
High & -.370 \\
Professional Training: None (Ref.) & \\
Apprenticeship, External Training, etc. & -.126 \\
University Degree, Master Craftsman & $-.623^{* * *}$ \\
Employment status before Unemployment: Employed (Ref.) & \\
Subsidized Employment & -.051 \\
School, Apprentice, Military, etc. & $0.328^{* *}$ \\
Maternity Leave & 0.208 \\
Other & -.033 \\
Unemployment Benefit Recipient (yes) & $0.552^{* * *}$ \\
Level of Unemployment benefits & -.00006 \\
Months in Unemployment (div. by age-18) & $-.089^{* * *}$ \\
Months in Employment (div. by age-18) & 0.003 \\
\hline Obs. & 7780 \\
Adjusted-R2 & 0.029 \\
log-Likelihood & -20782.65 \\
\hline \hline & \\
\hline
\end{tabular}

Note: Additional control variables used in the estimation: Months of entry into unemployment (June 2007 - April 2008) and time between entry and interview (in weeks). Full estimation results are available on request by the authors.

$* * * / * * / *$ indicate significance at the $1 \% / 5 \% / 10 \%$-level. 
Table A.3: OLS Estimation Results - Reservation Wage and Number of Search Channels

\begin{tabular}{|c|c|c|}
\hline & $\log \phi$ & $s$ \\
\hline \multicolumn{3}{|l|}{ ALMP Expectation: Low (0-4) } \\
\hline High $(5-10)$ & $-.027^{* * *}$ & $0.283^{* * *}$ \\
\hline West Germany & $0.169^{* * *}$ & $0.207^{* * *}$ \\
\hline Female & $-.124^{* * *}$ & 0.028 \\
\hline Internal Locus of Control & $0.029^{* * *}$ & 0.031 \\
\hline German citizenship & -.0002 & 0.127 \\
\hline Married (or cohabiting) & -.006 & $0.138^{* * *}$ \\
\hline \multicolumn{3}{|l|}{ No Children (Reference cat.) } \\
\hline One Child & $0.036^{* * *}$ & -.024 \\
\hline Two (or more) Children & $0.064^{* * *}$ & $-.223^{* * *}$ \\
\hline \multicolumn{3}{|l|}{ Age (17-24 years) } \\
\hline Age (25-34 years) & $0.093^{* * *}$ & $-.143^{* *}$ \\
\hline Age (35-44 years) & $0.138^{* * *}$ & -.056 \\
\hline Age (45-55 years) & $0.139^{* * *}$ & $-.146^{* *}$ \\
\hline \multicolumn{3}{|l|}{ School Leaving Degree: No degree (Ref.) } \\
\hline Low & $0.049^{* *}$ & 0.096 \\
\hline Medium & $0.068^{* * *}$ & $0.3^{* *}$ \\
\hline High & $0.155^{* * *}$ & 0.146 \\
\hline \multicolumn{3}{|l|}{ Professional Training: None (Ref.) } \\
\hline Apprenticeship, External Training, etc. & $0.073^{* * *}$ & $0.292^{* * *}$ \\
\hline University Degree, Master Craftsman & $0.233^{* * *}$ & $0.456^{* * *}$ \\
\hline \multicolumn{3}{|c|}{ Employment status before Unemployment: Employed (Ref.) } \\
\hline Subsidized Employment & -.015 & -.003 \\
\hline School, Apprentice, Military, etc. & $-.047^{* * *}$ & -.094 \\
\hline Maternity Leave & $-.031^{* *}$ & -.139 \\
\hline Other & -.012 & -.108 \\
\hline Unemployment Benefit Recipient (yes) & $-.052^{* * *}$ & -.044 \\
\hline Level of Unemployment Benefits & $0.013^{* * *}$ & $0.033^{* *}$ \\
\hline Months in Unemployment (div. by age-18) & $-.013^{* * *}$ & -.012 \\
\hline Months in Employment (div. by age-18) & $0.001^{* *}$ & $0.01^{* * *}$ \\
\hline Obs. & 7780 & 7780 \\
\hline Adjusted-R2 & 0.282 & 0.036 \\
\hline log-Likelihood & -737.267 & -14843.55 \\
\hline
\end{tabular}

Note: Additional control variables used in the estimation: Months of entry into unemployment (June 2007 - April 2008) and time between entry and interview (in weeks). Full estimation results are available on request by the authors.

$* * * / * * / *$ indicate significance at the $1 \% / 5 \% / 10 \%$-level. 
Table A.4: Propensity Score Estimation: General Participation Expectation in ALMP - High $(\eta=5-10)$ vs. Low $(\eta=0-4)$

\begin{tabular}{ll}
\hline \hline Variable & Coefficient \\
\hline West Germany & $0.274^{* * *}$ \\
Female & $0.156^{* * *}$ \\
Internal Locus of Control & 0.062 \\
German citizenship & $-.240^{* *}$ \\
Married (or cohabiting) & -.070 \\
No Children (Reference cat.) & \\
One Child & 0.021 \\
Two (or more) Children & 0.111 \\
Age (17-24 years) & \\
Age (25-34 years) & $-.219^{* * *}$ \\
$\quad$ Age (35-44 years) & $-.228^{* * *}$ \\
Age (45-55 years) & $-.403^{* * *}$ \\
School Degree: No degree (Ref.) & -.082 \\
Low & -.187 \\
Medium & $-.386^{* *}$ \\
High & \\
Professional Training: None (Ref.) & -.118 \\
Apprenticeship, External Training, etc. & $-.402^{* * *}$ \\
University Degree, Master Craftsman & \\
Employment status before Unemployment: Em- & \\
ployed (Ref.) & 0.026 \\
Subsidized Employment & $0.161^{*}$ \\
School, Apprentice, Military, etc. & 0.08 \\
Maternity Leave & -.031 \\
Other & $0.335^{* * *}$ \\
Lnemployment Benefit Recipient (yes) & -.008 \\
Months in Unemployment (div. by age-18) & $-.071^{* * *}$ \\
Months in Employment (div. by age-18) & -.002 \\
\hline R2 & 0.026 \\
Log-Likelihood & -5159.429 \\
Hit-rate & 57.40 \\
\hline \hline & \\
\hline &
\end{tabular}

Note: Estimations are done using a logit model. Additional control variables used: Months of entry into unemployment and time between entry and interview.

$* * * / * * / *$ indicate significance at the $1 \% / 5 \% / 10 \%$-level. 
Figure A.1: Propensity Score Distribution in Both Groups

High vs. Low Participation Expectation

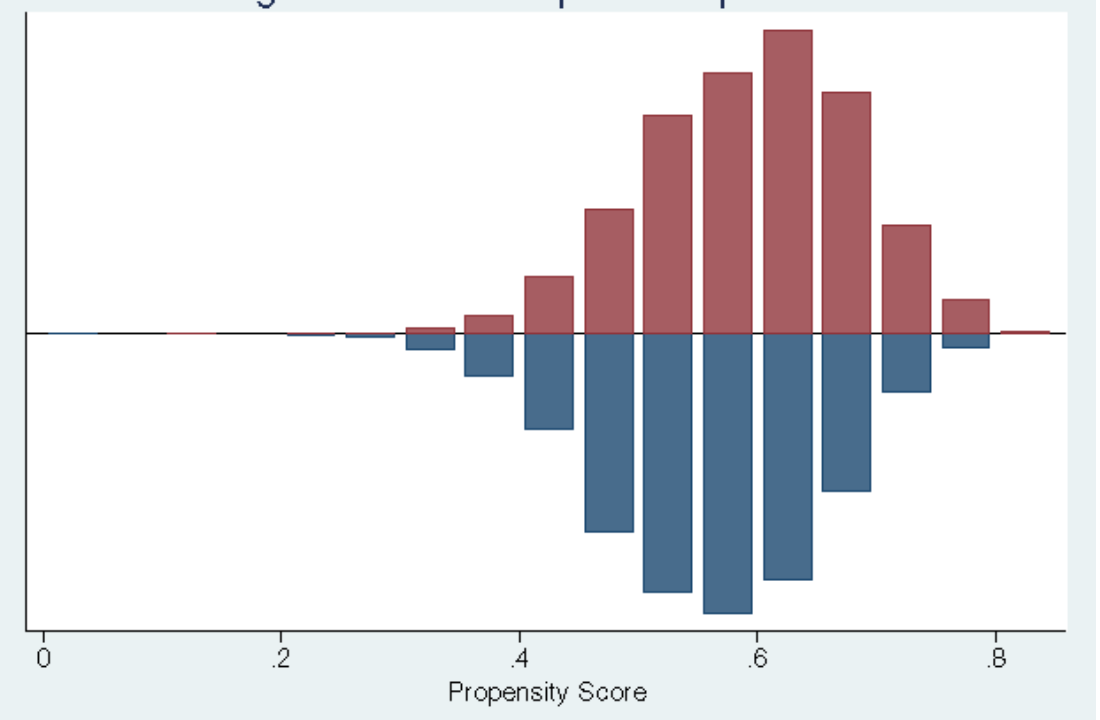

Note: Propensity score estimation results can be found in Table A.4. Individuals with high participation expectations $(\eta=5-10)$ are depicted in the upper half, individuals with low participation expectations $(\eta=$ $0-4)$ in the lower half. 